Hopf, N.B.; Ruder, A.; Waters, M.A.; Succop, P. Concentration-dependent halflives of polychlorinated biphenyl in sera from an occupational cohort. Chemosphere, 91(2):172-178, 2013.

\begin{tabular}{|l|l|}
\hline Postprint version & Final draft post-refereeing \\
\hline Journal website & $\underline{\text { http://www.sciencedirect.com/science/journal/00456535 }}$ \\
\hline Pubmed link & $\underline{\text { http://www.ncbi.nlm.nih.gov/pubmed/23336921 }}$ \\
\hline DOI & $\underline{10.1016 / \text { j.chemosphere.2012.12.039 }}$ \\
\hline
\end{tabular}




\title{
Concentration-dependent Half-Lives of Polychlorinated Biphenyl in Sera from an Occupational Cohort
}

\author{
Nancy B. Hopf ${ }^{1}$, Avima M. Ruder ${ }^{2}$, Martha A. Waters ${ }^{2}$, Paul Succop ${ }^{3}$ \\ ${ }^{1}$ Institute for Work and Health (IST), Rue du Bugnon 21, CH-1011 Lausanne, Switzerland. \\ Nancy.Hopf@hospvd.ch; NancyBHopf@gmail.com \\ ${ }^{2}$ Centers for Disease Control and Prevention (CDC), National Institute for Occupational Safety \\ and Health (NIOSH), 4676 Columbia Parkway, Cincinnati, Ohio 45226, USA. amr2@cdc.gov; \\ maw0@cdc.gov \\ ${ }^{3}$ University of Cincinnati, Department of Environmental Health, Kettering G29, PO Box \\ 670056, Cincinnati, Ohio 45267, USA. \\ succopa@uc.edu
}

Disclaimers: Mention of company names or products does not constitute endorsement by the National Institute for Occupational Safety and Health. The findings and conclusions in this report are those of the authors and do not necessarily represent the views of the National Institute for Occupational Safety and Health.

Corresponding author:

Nancy B. Hopf, Ph.D.

Institute for Work and Health (IST), Rue du Bugnon 21, CH-1011 Lausanne, Switzerland

Nancy.Hopf@hospvd.ch; NancyBHopf@gmail.com

+41 (0) 213149558 (voice) +41 (0) 213147430 (fax)

Keywords: Polychlorinated biphenyls, PCB, half-life, serum PCB

Highlights

1. This is the largest and longest studied cohort of workers exposed to PCBs for whom serum values are available

2. We present PCB half-life estimates using sera from four time points

3. PCB half-life estimates are for two commercial PCB mixtures (Aroclor 1254 and 1242)

4. Two serum PCB elimination rates were discovered (rapid and then slow)

5. Gender differences were observed only for Aroclor 1242 in earlier years. 
Polychlorinated biphenyls (PCBs) are carcinogenic. Estimating PCB half-life in the body based on levels in sera from exposed workers is complicated by the fact that occupational exposure to PCBs was to commercial PCB products (such as Aroclors 1242 and 1254) comprised of varying mixtures of PCB congeners. Half-lives were estimated using sera donated by 191 capacitor manufacturing plant workers in 1976 during PCB use (1946-1977), and post-exposure (1979, 1983, and 1988). Our aims were to: (1) determine the role of covariates such as gender on the half-life estimates, and (2) compare our results with other published half-life estimates based on exposed workers. All serum PCB levels were adjusted for PCB background levels. A linear spline model with a single knot was used to estimate two separate linear equations for the first two serum draws (Equation A) and the latter two (Equation B). Equation A gave half-life estimates of 1.74 years and 6.01 years for Aroclor 1242 and Aroclor 1254, respectively. Estimates were 21.83 years for Aroclor 1242 and 133.33 years for Aroclor 1254 using Equation B. High initial body burden was associated with rapid PCB elimination in workers at or shortly after the time they were occupationally exposed and slowed down considerably when the dose reached background PCB levels. These concentration-dependent half-life estimates had a transition-point of 138.57 and $34.78 \mathrm{ppb}$ for Aroclor 1242 and 1254, respectively. This result will help in understanding the toxicological and epidemiological impact of exposure to PCBs in 25 humans. 


\subsection{Introduction}

27 Many electrical equipment manufacturing workers in the United States were exposed to polychlorinated biphenyls (PCBs) before their use was banned in 1977. The National Institute

29 for Occupational Safety and Health (NIOSH) is conducting epidemiologic studies of the possible

30 health consequences of occupational exposure to PCBs at three capacitor manufacturing plants

31 (Prince et al., 2006; Rocheleau et al 2011; Ruder et al., 2006; Silver et al., 2009; Steenland et al., 32 2006), using job-exposure matrices as a surrogate for PCB body burden.

34 The PCB body burden (dose levels) over time depends upon toxicokinetics, which includes 35 elimination half-lives (Shirai and Kissel, 1996). Intrinsic human elimination half-lives for PCBs 36 were 10-15 years in a recent study (Ritter et al 2011), derived from cross-sectional biomonitoring 37 data. In humans with occupational exposure to PCBs, several studies have provided estimates for 38 PCB half-life varying from < 1 year to 71 years (Steele et al., 1986; Phillips et al., 1989; Taylor 39 and Lawrence, 1992; Wolff et al., 1992, Seegal et al., 2011). These previous half-life estimates

40 were usually based on only two measurements. The objectives of the current effort were to 41 develop estimates of the half-lives of commercial PCB mixtures Aroclor 1242 and Aroclor 1254 42 using all available sera from a select group of workers from one of the three plants, to consider 43 the role of high initial body burden and gender on the half-life estimates, and to compare the 44 results with other published half-life estimates based on occupational exposure. The estimates 45 were adjusted for PCB background levels (Hopf et al 2009). This is the only study that 46 incorporates four PCB blood measurements over time, allowing us to explore PCB elimination 47 rates. 


\section{$50 \quad 2.0$ Background}

51 Shirai and Kissel (1996) reviewed fourteen studies of humans with occupational, accidental, or

52 experimental exposure to PCBs and concluded that very short (i.e., < 1 year) and very long (i.e,

$53>10$ years) half-lives were probably unlikely. The exponential decay model, assuming first-

54 order kinetics, is given by

$$
X(t)=X\left(t_{0}\right) e^{-\lambda\left(t-t_{0}\right)}
$$

56 where $\mathrm{X}(\mathrm{t})$ is the concentration in the compartment at time $\mathrm{t}, \mathrm{X}\left(\mathrm{t}_{0}\right)$ is the unknown initial

57 concentration at time $t_{0}$, and $\lambda$ is the elimination constant which has units of time ${ }^{-1}$. The half-life,

$58 t_{1 / 2}$, is defined as the length of time after which the initial concentration of the chemical is

59 reduced by half, and is equal to the natural logarithm of 2 divided by the elimination constant, or

$60 \mathrm{t}_{1 / 2}=\ln (2) / \lambda$ (Cassarett and Doull, 1986).

61

62 In a controlled pharmacokinetic study, the initial concentration of a chemical in a compartment is

63 known and subsequent concentrations are measured at specific time points. In occupational

64 studies, the initial concentration of a chemical in a compartment after first exposure is usually

65 unknown. Also, exposure typically is chronic, not acute, occurring at different rates over

66 different periods of time. However, measurements taken after exposure is known to have ceased

67 can be used to estimate the half-life for elimination of the chemical from the sera compartment.

69 Estimating the half-life of PCBs in the body using sera from exposed workers is complicated;

70 occupational exposure to PCBs was generally to commercial PCB products comprised of varying

71 mixtures of some of the 209 PCB congeners. Different methods exist for quantifying the level of

72 PCBs in sera (Lawton et al., 1985a). Ideally, congener-specific serum PCB levels would be 
73 measured. However, in the past, results were typically either quantified using an “Aroclor

74 standard” by summing the heights of selected peaks on the gas chromatogram (GC) which

75 correlated with hallmark peaks in a specific Aroclor, or quantified as lower- or higher-

76 chlorinated PCBs (LPCBs or HPCBs) based on the sum of the GC peaks that occurred before or

77 after the retention time of p,p'-dichlorodiphenyldichloroethylene (DDE), universally detected in

78 sera from people in the industrialized world. The two methods of quantifying PCBs in sera are

79 related, with 93\% of Aroclor 1242 being LPCBs and 96\% of Aroclor 1260 being HPCBs

80 (Lawton et al., 1985b). The range of chlorination of the Aroclors in sera was determined to be

81 Aroclor 1016: 2-5 chlorines, Aroclor 1242: 2-6 chlorines, Aroclor 1254: 4-7 chlorines, and

82 Aroclor 1260: 4-8 chlorines (Lawton et al., 1985b).

84 In addition to degree of chlorination, several other factors are considered to be related to the half-

85 life of PCBs in humans. Wolff et al. (1992) found PCBs in serum from workers (N=60) in jobs

86 with high and direct PCB exposure had shorter half-lives for lower chlorinated PCBs than PCBs

87 in serum from workers $(\mathrm{N}=105)$ in jobs with low and indirect PCB exposure (4.8 versus 17

88 years, respectively). A follow-up study of some of these workers $(\mathrm{N}=45) 28$ years later (Seegal

89 et al., 2011), showed somewhat longer half-lives than Wolff et al. (1992) had, but showed the

90 same trend with respect to high initial PCB body burden: half-life estimates for lower chlorinated

91 PCBs were 13.5 and 7.3 years and for higher chlorinated PCBs were 32.6 and 12 years for low

92 and high exposed workers, respectively. Factors complicating the estimation of PCB half-life

93 include percent body fat, laboratory measurement error (Caudill et al., 1992), the time interval

94 between measurements (Caudill et al., 1992), and continuing low-level exposure to PCBs (Shirai

95 and Kissel, 1996), such as background levels of exposure (Michalek et al., 1998). 
97 Different statistical methods have been proposed for determining the half-life of a substance in

98 humans from an exposed population. The choice of method often depends on the number of 99 participants and the number of measurements available for each participant over time. Steele et

100 al. (1986) used linear regression to estimate the half-lives of PCBs in the body using sera

101 quantified as Aroclors 1242 and 1260. Five capacitor manufacturing workers at one of the

102 NIOSH cohort plants, selected because of their high probability of PCB exposure, provided sera

103 at two draws separated by 84 months. The analysis produced half-life estimates of 2.0 years for

104 Aroclor 1242, 27.6 years for Aroclor 1260, and 11.5 years for total serum PCBs. The median

105 technique was developed by Phillips et al. (1989) to estimate the half-lives of Aroclors 1242 and

1061254 for 60 former capacitor manufacturing workers (at the same NIOSH cohort plant) with two

107 serum draws separated by 100 months. Phillips et al. reported half-life estimates of 2.6 years for

108 Aroclor 1242 and 4.8 years for Aroclor 1254. In addition, they observed a decreasing trend in

109 half-life based on initial concentration of PCBs in 1977, although Shirai and Kissel (1996)

110 showed that this was likely due to confounding by continuing exposure to PCBs. Table 1 gives

111 an overview of previous half-life estimates from US occupational exposed populations and type

112 of statistical methods used to derive these half-lives.

113

114 Lawton et al. (1985b) initiated a research study in 1976 to study the possible health effects of

115 PCB exposure in workers at another capacitor manufacturing plant (also in the NIOSH cohort)

116 with two facilities in upstate New York. Aroclor 1254 was used at this plant from 1946-1954,

117 Aroclor 1242 from 1954-1971, and Aroclor 1016 from 1971-1977 (Lawton et al., 1985b). In

118 1976, 194 employees, selected because their jobs "required direct contact with PCB in zones of 
119 high air concentration, were in the immediate periphery of the high exposure zone, or had high

120 but intermittent exposure”, underwent examinations that included the collection of blood sera

121 from most $(\mathrm{n}=191)$. This first examination was approximately one year prior to the termination

122 of PCB use at the plant. Serum draws were repeated in 1979, 1983, and 1988 for subsets of the

123191 participants. Taylor et al. (1992) used measured serum PCB levels from 148 of the workers

124 who provided sera in both 1979 and 1983 to produce half-life estimates of 1.8 years (95\%

125 confidence interval $(\mathrm{CI})=1.7-1.9)$ for Aroclor 1242 , 3.3 years $(95 \% \mathrm{CI}=3.0-3.8)$ for Aroclor

126 1254, and 4.1 years $(95 \% \mathrm{CI}=3.6-4.7)$ for Aroclor 1260.

127

$128 \quad 3.0$ Materials and Methods

129 The basis for the Aroclor half-life estimates were the Aroclor 1242, 1254, and 1260 serum levels

130 calculated by Lawton et al. (1985). According to study plant records, the use of PCBs in the two

131 facilities ended 30 June 1977. Time intervals from last exposure were measured from that date.

132 Specific dates of the serum draws were not known, but estimated as 15 March 1976, 15

133 November 1979, 15 November 1983, and 1 November 1988 since it is known that PCBs were

134 "still in use during the first 1.3 years after the first examination” (Brown et al., 1989); the second

135 serum draw was in November 1979 (Brown et al., 1984); the third serum draw was 48 months

136 after the second (Taylor et al., 1992); and the fourth serum draw occurred in 1988.

137

138 A majority of the 191 participants in the Lawton study provided sera at four time points

139 (122/194, 63\%). Thirty-five participants provided sera at three time points, 19 at two, 15 at one,

140 and three did not provide sera at any of the time points. Therefore, our statistical analysis was

141 performed using results of 191 workers. PCB serum levels were quantified for 185 participants 
142 in 1976, 173 in 1979, 150 in 1983, and 138 in 1989. Most participants were male (152/194, $14378 \%)$.

145 The methods for quantifying PCB levels (packed column GC with electron capture detector) in 146 the serum have been described (Lawton et al., 1985a). Aroclor standards were used to calculate 147 PCB levels in terms of “Aroclor 1242” and “Aroclor 1254”. Serum PCB levels were quantified 148 by summing peak heights with relative retention times of 37, 70, and 84 as Aroclor 1242 and 149 with relative retention times of 125, 146, 160, and 184, as described in Lawton et al (1985a) as 150 Aroclor 1254 (Brown et al., 1989). (Peak 184 differed from the relative retention time of 174 151 described in the Webb-McCall method (Webb and McCall, 1973).

153 Although PCB exposure was ongoing at the time of the first serum measurement, it might be 154 possible to use data from the 1976 serum draw to estimate the half-life for Aroclor 1254 (Brown 155 et al., 1989) since Aroclor 1254 was only used from 1946-1954 at the study plant (Lawton et al., 156 1985b). Other than gender, information on factors known to affect half-life such as age and

157 percent body fat was unavailable. All statistical analyses were performed using SAS 9 Software 158 (SAS Institute Inc., Cary, NC).

160 Background PCB levels might contribute to the length of the half-life estimates; therefore an 161 adjustment for PCB background levels for the years 1976, 1979, 1983, and 1988 was needed. 162 No national statistically based cross-sectional data concerning historical serum PCB levels exist 163 for the U.S. population by year. Therefore, we estimated the background PCB serum levels for 
164 these four time points based on the scientific literature (Hopf et al., 2009). The background PCB

165 levels were estimated to follow negative exponential "die away" curves;

- $\quad$ for Aroclor 1242; corr_pcb = pcb - 4.18*exp(-0.088*(year-1977));

- $\quad$ for Aroclor 1254; corr_pcb = pcb - 8.5788*exp(-0.0569*(year-1977));

168 where corr_pcb represents the serum PCB levels (ppb) corrected for background, and pcb is the

169 uncorrected values. Adjustments for PCBs background serum levels were performed by

170 subtracting the estimated background population serum PCB concentrations from the measured

171 serum PCB concentrations prior to log transformation.

172

173 Quantile-quantile plots of natural-log-transformed serum PCB levels (ln(PCB)) versus standard

174 normal quantiles indicated that the data were lognormally distributed (not shown). Figures 1 and

1752 depict the individual profiles of the log-transformed serum PCB levels over time, for Aroclors

1761242 and 1254, respectively. Vertical reference lines indicate the times of the serum draws

177 relative to the end of PCB use, and average trend lines were fit using a smoothing routine for

178 females and males. The quadratic term for time was statistically significant for fitting a linear

179 model for all years, indicating that the assumptions of a first-order kinetic model were violated.

180 From the graphs it was apparent that the decrease in earlier years was faster than in later years.

181 This bi-phasic elimination was consistent with elimination patterns from high initial body burden

182 of other chlorinated persistent chemicals such as TCDD (Aylward et al. 2005). Therefore, a

183 linear spline model with a single knot (Keele 2008; Altshuler 1981) approach was used to

184 demonstrate that there were two slopes underlying the die-away curve. This model fit the data

185 well; a linear equation was fitted for the 1976 and 1979 data (Equation A), and a second linear

186 equation was used to fit the 1983 and 1988 data (Equation B) using PROC MIXED. The equality 
187 of the slopes estimated over the two time periods was tested by analyzing all the data in a single

188 model which included the interaction between periods with slope. The dependent variables were

189 the natural log transformed PCB Aroclors $\ln (\mathrm{PCB})$.

191 The point (transition point) where Equation A and Equation B intersected was estimated by

192 determining the year during which the two regression equations for equations A and B provided

193 the same estimate for PCB concentrations. Other parameters besides background PCB levels

194 affecting elimination such as additional elimination mechanisms (PCB fecal elimination of

195 unchanged compound through lipid partitioning into the large intestine) were unknown and could

196 not be included in the model; hence our model estimates half-life elimination rates based on

197 PCBs (not metabolites) circulating in the blood. The transition point indicating a possible change 198 in elimination rate from rapid to slow was calculated.

\section{$200 \quad 4.0$ Results}

201 Aroclor 1242 quantified measurements increased in five participants from 1976 to 1979, in four 202 participants from 1979 to 1983, and in 32 participants from 1983 to 1988. Likewise, Aroclor 2031254 quantified measurements increased in 49 participants from 1976 to 1979, in 16 participants 204 from 1979 to 1983, and in 50 participants from 1983 to 1988.

205

206 Detailed work histories, available for 192 of the 194 participants, described 2,684 years of 207 employment at the study plant during the era of PCB exposure (January 1, 1946 - June 30, 208 1977). The median year first employed was 1965 (range 1946 - 1974) and the median duration 209 of employment was 11 years (range 1 - 30 years). 
211 Summary statistics for the measured serum PCB levels, quantified as Aroclors 1242 and 1254 in

212 parts per billion (ppb) with and without corrections for background serum PCB levels, are

213 provided in Table 2. Measured serum PCB levels were highly variable within a given year. The

214 difference between corrected and uncorrected serum PCB levels was minimal in earlier years due

215 to the high serum PCB levels (e.g. 1976: GM was 1,521 ppb) compared to the value used to

216 adjust for background levels.

218 The year Equation A and Equation B intersected (transition point) for Aroclor 1242 was 1980

219 and for Aroclor 1254, 1982. We did not find a statistical significant difference in half-life

220 estimates between genders for Aroclor 1254. This was also true for Aroclor 1242 in later years

221 (Equation B). However, the Aroclor 1242 half-life estimate for equation A (1976-1979) was

222 significantly longer in females (half-life of 2.15 years, 95\% confidence interval [C.I.] 1.7 - 2.9

223 years) as compared to males (1.7 years, 95\% C.I. $1.4-2.2$ years, $\mathrm{t}=2.21, \mathrm{p}<0.03$ ).

225 The transition point concentration for Aroclor 1242 and 1254 were 138.57 and 34.78 ppb, 226 respectively. The elimination kinetics of PCBs were concentration dependent with faster rates 227 observed at higher concentrations (>138.57 ppb for Aroclor 1242 and >34.78 ppb for Aroclor $2281254 \mathrm{ppb}$ ) and the transition to slower rates occurring below this concentration.

230 Results of the repeated measures regression models and subsequent half-life estimates are

231 provided in Table 3. In the linear spline model with a single knot, the first slope using the first 232 two serum draws (Equation A) produced an estimated half-life of 1.74 years for Aroclor 1242 
$233(95 \% \mathrm{CI}=1.60-1.89$ years $)$ and 6.01 years for Aroclor $1254(95 \% \mathrm{CI}=4.53-8.93$ years $)$.

234 Using the last two serum draws (Equation B) to model the second slope produced an estimated

235 half-life of 21.83 years for Aroclor 1242 (95\% CI = $16.92-30.76$ years) and 133.33 years for

236 Aroclor 1254 (95\% CI = $52.88-\infty$ years). The slopes estimated for the two time periods were

237 significantly different $(\mathrm{p}<0.0001)$.

238

239 We compared the geometric means (cGM) of predicted values of Aroclor 1242 with actual cGM

240 for 1976 (predicted 1503: actual 1528), 1979 (predicted 274: actual 280), 1983 (predicted 115:

241 actual 125), and 1988 (predicted 91: actual 92); and for Aroclor 1254: 1976 (predicted 78: actual

242 85), 1979 (predicted 48: actual 55); 1983 (predicted 28: actual 35), and 1988 (predicted 27:

243 actual 32).

$245 \quad 5.0$ Discussion

246 Estimates of the half-lives of PCBs were based on sera drawn from the same individuals at up to

247 four time points. The half-life, defined as the change in concentration in the body over time is

248 the net result of elimination from the body, changes in the body composition, and intake from the

249 environment. Factors affecting elimination are age, smoking, initial body burden, body fat, and

250 gender (Milbrath et al 2009). Although the gender of each participant was known, age and body

251 mass index data were not available; therefore, it was not possible to adjust for covariates other

252 than gender.

253

254 Half-life estimates for sera from female participants were generally longer than half-life

255 estimates for sera from male participants, although the gender difference was only statistically 
256 significant for Aroclor 1242 in the earlier years (equation A). This gender difference in half-life

257 estimates was also seen in Seegal et al., (2011) not only in lower but also higher chlorinated

258 PCBs. PCBs are known to be stored in adipose tissue, therefore half-life estimates are

259 potentially confounded by percent body fat in addition to the rate of PCB migration to the blood

260 (Brown, 1994) or hormonal differences (Kang et al,. 2008; Cerna et al., 2008). No clear

261 explanations for this gender-related difference exist, and further investigation is needed.

262

263 High initial PCB body-burden showed a concentration-dependent biphasic elimination rate,

264 which has been identified in related compounds such as 2,3,7,8-tetrachloro-p-dibenzo-dioxin

265 (TCDD) and penta-chlorodibenzofuran (PeCDF) in cases of acute poisoning (Abraham et al

266 2002), Seveso incident (Aylward et al 2005; Michalek et al 2002) in children (Kerger et al 2006),

267 Yusho and Yu-Cheng rice oil poisoning (Leung et al 2007; Ryan et al 1993), but has until now

268 not been reported in workers with chronic high exposures. This non-linear behavior might be due

269 to possible enzyme induction similarly to that seen in TCDD and pentaCDF, as enzyme

270 induction has been observed previously in animals exposed to PCBs (Emond et al, 2005).

272 Not adjusting for background PCB levels did not have an impact on equation A (half-lives not 273 corrected for background levels: Aroclor 1242: 1.75 years and Aroclor 1254: 6.8 years). The

274 half-life estimates for uncorrected serum PCB levels using equation B were shortened; giving 275 Aroclor 1242 an half-life of 20.4 years and statistically significantly shortened half-life for 276 Aroclor 1254 of 69.5 years. Since most of these individuals continued to live in the vicinity of 277 the plant they would have an ongoing environmental exposure as would eating contaminated fish 
278 in the 1980s. Accounting for background PCB levels will give a more realistic estimate of the 279 half-life.

281 Serum levels were reduced by approximately $90 \%$ and $50 \%$ between the first and third serum 282 draws for Aroclors 1242 and 1254, respectively, but increased between the third and fourth 283 serum draws for $23 \%$ and $36 \%$ of the participants. By the fourth serum draw, serum PCB levels 284 had fallen to much lower levels and were possibly approaching the levels of those in the general 285 population who have somewhat elevated environmental exposures. The reasons for the increases 286 in serum PCB levels between consecutive serum draws seen in some workers are not known. 287 Possible explanations are de-chlorination of congeners to lower chlorinated PCB; where 288 particular congeners would be quantified as Aroclor 1254 in earlier serum draws, and as Aroclor 2891242 in later serum draws. Continued occupational exposures were possible for those workers 290 who dismantled the plant, and as mentioned above living in the vicinity could give higher 291 environmental exposures. Other factors that could lead to increases in PCBs in these workers are 292 laboratory measurement error, increased release of PCBs into the blood from body fat storage, or 293 a combination of factors. These factors could lead to unrealistic long half-life estimates.

295 Dioxin-like (DL) PCBs (PCB77, PCB 81, PCB 126, PCB 169, PCB 105, PCB 114, PCB 118, 296 PCB 123, PCB 156, PCB 157, PCB 167, PCB 89) are tetra-, penta-, and hexa-chlorinated 297 congeners. A complete PCB congener distribution of Aroclor mixtures has been published 298 (Frame et al 1996), and the weight\% of DL-PCB congeners for Aroclor 1016, Aroclor 1242, and 299 Aroclor 1254 were $0 \%, 1.95 \%$, and $23.58 \%$, respectively. Therefore workers in the early time 300 period exposed to Aroclor 1254 (before the 1960s) had higher DL-PCB exposures compared to 
301 workers who were only exposed to Aroclor 1242 ( 1960-1970) and Aroclor 1016 (in the 1970s)

302 used in later time periods. DL-PCBs in workers' blood samples can be estimated from Seegal et

303 al 2011; they re-analyzed stored blood samples previously determined as lower (LPCBs; peaks

304 eluding before DDE in the chromatogram) and higher (HPCBs; peaks eluding after DDE in the

305 chromatogram) PCBs, and found four DL-PCBs congeners (118, 105, 167, 156); all present in

306 peaks quantified as HPCBs.

307

308 Shirai and Kessel (1996) reasoned that half-lives longer than 10 years were probably the result of

309 confounding by additional exposure. Wolff et al. (1992), on the other hand, observed long half-

310 lives which they attributed to the presence of congeners with relatively longer half-lives in the

311 sera compared to the distribution of congeners in the Aroclor mixtures. The explanation we can

312 offer is that elimination of PCB congeners with shorter half-lives (generally, lower chlorinated

313 biphenyls) from high initial PCB serum levels is estimated with Equation A, while Equation B is

314 an estimate of the elimination of PCB congeners with long half-life as described by Wolff et al

315 (1992) with continuous exposure to PCB background levels as described by Shirai and Kessel

316 (1996). Researchers who have continuously monitored PeCDF in a group of Yusho patients,

317 poisoned by PCB contaminated rice bran oil, have recently reported that these patients maintain

318 a high level resulting in an infinite half-life (Matsumoto et al 2009). The authors suggested that a

319 more complicated model to explain excretion in humans were needed.

321 One of the strengths of this study was that PCB measurements were available at up to four time

322 points per participant, and a linear spline model with a single knot was used to estimate PCB

323 half-lives. Advantages of the linear spline model with a single knot include generalizability to 
324 any number of repeated measures, the ability to handle varying numbers of repeated measures

325 per participant, and the ability to adjust the half-life estimate for covariates such as gender by

326 adding the appropriate terms and interactions to the model.

328 Unlike all previous half-life estimates reported in the scientific literature, ours is based on serum 329 draws from four time points, which allowed us to explore more than one linear regression PCB 330 elimination rate. A single linear regression using all four time points did not achieve as good a

331 fit as did the linear spline model with a single knot. We did not try more than a single knot as

332 based on the data; the linear slope for decline appeared much steeper between the first 2

333 occasions than the latter two. This bi-phasic decay was also observed by Brown and Lawton

334 (2001) who tried to fit a pseudo-first order elimination to their studies' three serum draws. Other 335 studies (Phillips et al., 1989; Taylor and Lawrence, 1992; Brown et al., 1989; Wolff et al., 1992)

336 estimated half-lives based on serum draws at only two time points, which resulted in shorter half-

337 lives (Table 1). The half-life estimates using the linear spline model with a single knot were

338 somewhat shorter for Equation A and longer for Equation B than estimates previously published,

339 because it incorporates high initial PCB body burden (Equation A) and low body burden

340 (Equation B). The equation A estimated half-life of 1.8 years for Aroclor 1242 was 31\% shorter

341 than the 2.6-year estimate by Phillips (1989), 81\% shorter than the 9.6-year estimate by Seegal et

342 al. (2011), but the same as the estimate by Taylor et al. (1992). Equation B estimated an half-life

343 of 21.8 years for Aroclor 1242, 2.3, 7.4, and 11.1 times longer than the estimates by Seegal et al.

344 (2011), Phillips (1989), and Taylor et al. (1992), respectively. Likewise, Equation A estimated a 345 half-life of 6.01 years for Aroclor 1254, which was 42 and 106\% longer than the estimates by 346 Phillips (1989) and Taylor et al. (1992), respectively; and 90 and 62\% shorter than the estimates 
347 by Wolff et al (1992) and Seegal et al (2011). Equation B estimated half-life of 133.33 years for

348 Aroclor 1254 was 27, 40, 1, and 7.5 times longer than estimates by Phillips (1989), Taylor et al.

349 (1992), Wolff et al. (1992), and Seegal et al. (2011), respectively. The half-life estimates using

350 the linear spline model with a single knot were consistent with elimination rates computed by

351 Phillips et al., 1989; Taylor and Lawrence, 1992; Brown et al., 1989 for the fast elimination rate

352 (Equation A) for a high internal dose, and by Wolff et al 1992, Shirai and Kissel 1996, and

353 Seegal et al. 2011 for the slow elimination rates (Equation B) for a low internal dose approaching

354 background PCB levels.

355

356 Major limitations of this study include not being able to estimate PCB congener-specific half-

357 lives because congener-specific serum data were not available. The PCB half-life estimates

358 would have improved if we could have adjusted for body mass index and age that are known to

359 affect PCBs half-lives. Strengths of these half-life estimates include a relatively long 13-year

360 sampling period, a large study population (191 participants), one to four serum draws per

361 participants, and three sampling points after occupational exposure ended. In addition, these

362 half-life estimates are for individuals with high initial body burdens, which we show here result

363 in concentration-dependent PCB half-lives. Attempts to back-calculate body burden in highly

364 exposed workers based on assumption of uniform half-lives will greatly underestimate dose and

365 overestimate potency as shown for TCDD (Aylward et al 2005).

\section{$367 \quad 6.0$ Conclusion}

368 Our PCB half-life estimates using the linear spline model with a single knot take into account 369 high initial body burden, ongoing environmental exposure, low serum levels, and congeners with 
370 very long half-lives. The estimated half-lives during a period of high internal dose were 1.74

371 years for Aroclor 1242 and 6.01 years for Aroclor 1254, modeling elimination using sera from

372 the first two serum draws. Half-lives during a period of low internal dose were estimated to be

37321.83 years and 133.33 years for Aroclor 1242 and Aroclor 1254, respectively, using the final

374 two serum draws. The transition point for Aroclor 1242 was $138.57 \mathrm{ppb}$ and for Aroclor 1254

375 was $34.78 \mathrm{ppb}$. The high internal dose estimates are in general agreement with the half-life

376 estimates reported in Phillips et al. (1989) and Brown and Lawton (2001), and the low dose

377 estimates are in agreement with Wolff et al. 1992 and Seegal et al. 2011. These half-life

378 estimates may aid in assessing PCB exposures retrospectively and reconstructing PCB dose

379 estimates for persons on whom biological monitoring was performed only once, which may in

380 turn help in understanding the toxicological and epidemiological impact of exposure to PCBs in 381 humans.

383 Acknowledgement: We thank all external, internal, and tri-partite reviewers for carefully 384 reviewing the manuscript. 


\section{References}

388

389

390

391

392

393

394

395

396

397

398

399

400

401

402

403

404

405

406

407

408

409

410

411

412

413

414

415

416

417

418

419

420

421

422

423

424

425

426

427

428

429

430

431

Abraham, K., Geusau, A., Tosun, Y., Helge, H., Bauer, S., Brockmoller, J., 2002. Severe 2,3,7,8tetrachlorodibenzo-p-dioxin (TCDD) intoxication: insights into the measurement of hepatic cytochrome P450 1A2 induction. Clin. Pharmacol. Ther. 72, 163-174.

Altshuler, B., 1981. Modeling of dose-response relationships. Environ. Health. Perspect. 42, 237.

Aylward, L.L., Brunet, R.C., Carrier, G., Hays, S.M., Cushing, C.A., Needham, L.L., Patterson, D.G. Jr., Gerthoux, P.M., Brambilla, P., Mocarelli, P., 2005. Concentration-dependent TCCD elimination kinetics in humans: toxicokinetic modeling for moderately to highly exposed adults from Seveso, Italy, and Vienna, Austria, and impact on dose estimates for the NIOSH cohort. J. Expo. Anal. Environ. Epidemiol. 15, 51-65.

Brown, D.P., 1987. Mortality of workers exposed to polychlorinated biphenyls-an update. Arch. Environ. Health. 42, 333-339.

Brown, J.F. Jr., Lawton, R.W., 1984. Polychlorinated biphenyl (PCB) partitioning between adipose tissue and serum. Bull. Environ. Contam. Toxicol. 33, 277-80.

Brown, J.F. Jr., Lawton, R.W., Ross, M.R., Feingold, J., Wagner, R.E., Hamilton, S.B., 1989. Persistence of PCB congeners in capacitor workers and yusho patients. Chemosphere. 19, 82934.

Brown, J.F. Jr., 1994. Determination of PCB metabolic, excretion, and accumulation rates for use as indicators of biological response and relative risk. Environ. Sci. Technol. 28, 2295-305.

Brown, J.F., Lawton, R.W., 2001. Factors controlling the distribution and levels of PCBs after occupational exposure. In Robertson and Hansen eds. PCBs. The University Press of Kentucky, 103-109.

Caudill, S.P., Pirkle, J.L., Michalek, J.E., 1992. Effects of measurement error on estimating biological half-life. J. Exp. Anal. Environ. Epi. 2, 463-476.

Cerna, M., Maly, M., Grabic, R., Batariova, A., Smid, J., Benes, B. 2008. Serum concentrations of indicator PCB congeners in the Czech adult population. Chemosphere 72, 1124-1131.

Emond, C., Charbonneau, M., Krishnan, K., 2005. Physiologically based modeling of the accumulation in plasma and tissue lipids of a mixture of PCB congeners in female spraguedawley rats. J. Toxicol. Environ. Health A. 68, 1393-1412.

Frame, G.M., Cochran, J.W., Bowadt, S.S., 1996. Complete PCB Congener Distributions for 17 Aroclor Mixtures Determined by 3 HRGC Systems Optimized for Comprehensive, Quantitative, Congener-Specific Analysis. J. High Resol. Chromatogr. 19, 657-668. 
Hopf, N.B., Ruder, A.M., Succop, P., 2009. Background levels of polychlorinated biphenyls in the U.S. population. Sci. Tot. Environ. 407, 6109-6119.

Kang, J.H., Park, H., Chang, Y.S., Choi, J.W., 2008. Distribution of organochlorine pesticides (OCPs) and polychlorinated biphenyls (PCBs) in human serum from urban areas in Korea. Chemosphere. 73, 1625-1631.

Keele, L. J. 2008. Semiparametric Regression for the Social Sciences. John Wiley \& Sons, Ltd, p. 49.

Kerger, B.D., Leung, H.W., Scott, P., Paustenbach, D.J., Needham, L.L., Patterson, D.G. Jr, Gerthoux, P.M., Mocarelli, P., 2006. Age-and concentration-dependent elimination half-life of 2,3,7,8-tetrachlorodibenzo-p-dioxin in Seveso children. Environ. Health. Perspec. 114, 15961602 .

Lawton, R.W., Brown, J.F. Jr., Ross, M.R., Feingold, J., 1985a. Comparability and precision of serum PCB measurements. Arch. Environ. Health. 40, 29-37.

Lawton, R.W., Ross, M.R., Feingold, J., Brown, J.F. Jr., 1985b. Effects of PCB exposure on biochemical and hematological findings in capacitor workers. Environ. Health. Perspec. 60, 165-184.

Leung, H.W., Kerger, B.D., Paustenbach, D.J., Ryan, J.J., Masuda, Y., 2007. Concentration and age-dependent elimination kinetics of polychlorinated dibenzofurans in Yucheng and Yusho patients. Toxicol. Ind. Health. 7, 493-501.

Matsumoto, S., Akahane, M., Kanagawa, Y., Koike, S., Yoshimura, T., Mitoma, C., Shibata, S., Uchi, H., Furue, M., Imamura, T., 2009. Variation in half-life of penta-chlorodibenzofuran (PeCDF) blood level among Yusho patients. Chemosphere. 77, 658-662.

Michalek, J.E., Pirkle, J.L., Needham, L.L., Patterson, D.G. Jr, Caudill, S.P., Tripathi, R.C., Mocarelli, P., 2002. Pharmacokinetics of 2,3,7,8-tetrachlorodibenzo-p-dioxin in Seveso adults and veterans of operation Ranch Hand. J. Expo. Anal. Environ. Epidemiol. 12, 44-53.

Michalek, J.E., Tripathi, R.C., Kulkarni, P.M., Gupta, P.L., Selvavel, K. 1998. Correction for bias introduced by truncation in pharmacokinetic studies of environmental contaminants. Environmetrics. 9, 165-174.

Milbrath, O.M., Wenger, Y., Chang, C.W., Emond, C., Garabrant, D., Gillespie, B.W., Jolliet, O., 2009. half-lives of dioxins, furans, and polychlorinated biphenyls as a function of age, body fat, smoking status and breast-feeding. Environ. Health. Perspec. 117, 417-425.

Phillips, D.L., 1989. Propagation of error and bias in half-life estimates based on two measurements. Arch. Environ. Contam. Toxicol. 18, 508-514. 
Phillips, D.L., Smith, A.B., Burse, V.W., Steele, G.K., Needham, L.L., Hannon, W.H., 1989. Half-life of polychlorinated biphenyls in occupationally-exposed workers. Arch. Environ. Health. 44, 351-354.

Prince, M.M., Ruder, A.M., Hein, M.J., Waters, M.A., Whelan, E.A., Nilsen, N., Ward, E.M., Schnorr, T.M., Laber, P.A., Davis-King, K.E., 2006. Mortality and exposure response among 14,458 electrical capacitor manufacturing workers exposed to polychlorinated biphenyls (PCBs). Environ. Health. Perspec. 114, 1508-1514.

Ritter, R., Scheringer, M., MacLeod, M., Moeckel, C., Jones, K.C., Hungerbühler, K., 2011. Intrinsic human elimination half-lives of polychlorinated biphenyls derived from the temrporal evolution of cross-sectional biomonitoring data from the United Kingdom. Environ. Health. Perspec. 119, 225-231.

Rocheleau CM, Bertke SJ, Deddens JA, Ruder AM, Lawson CC, Waters MA, et al. 2011. Maternal exposure to polychlorinated biphenyls and the secondary sex ratio: an occupational cohort study. Environ Health 10: 20.

Ruder, A.M., Hein, M.J., Nilsen, N., Waters, M.A., Laber, P., Davis-King, K., Prince MM, Whelan E., 2006. Mortality among workers exposed to polychlorinated biphenyls (PCBs) in an electrical capacitor manufacturing plant in Indiana: an update. Environ. Health. Perspec. 114, 1823.

Ryan, J.J., Levesque, D., Panopio, L.G., Sun, W.F., Masuda, Y., Kuroki, H., 1993. Elimination of polychlorinated dibenzofurans (PCDFs) and polychlorinated biphenyls (PCBs) from human blood in the Yusho and Yu-Cheng rice oil poisonings. Arch. Environ. Contam. Toxicol. 24, 504512.

SAS Institute Inc., SAS/STAT 9.1 User’s Guide. Cary, NC: SAS Institute Inc., 2004.

Seegal, R.F., Fitzgerald, E.F., Hills, E.A., Wolff, M.S., Haase, R.F., Todd, A.C., Parsons, P., Molho, E.S., Higgins, D.S., Factor, S.A., Marek, K.L., Seibyl, J.P., Jennings, D.L., McCaffrey, R.J., 2011. Estimating the half-lives of PCB congeners in former capacitor workers measured over a 28-year interval. J. Exp. Sci. Environ. Epi. 21, 234-246.

Shirai, J.H., Kissel, J.C., 1996. Uncertainty in estimated half-lives of PCBs in humans: impact on exposure assessment. Sci. Tot. Environ. 187, 199-210.

Silver, S.R., Whelan, E.A., Deddens, J.A., Steenland, N.K., Hopf, N.B., Waters, M.A. Ruder, A.M., Prince, M.M., Yong, L.C., Hein, M.J., Ward, E.M., 2009. Occupational exposure to polychlorinated biphenyls and risk of breast cancer Environ. Health. Perspect. 117, 276-282.

Steele, G., Stehr-Green, P., Welty, E., 1986. Estimates of the biologic half-life of polychlorinated biphenyls in human serum. New. Engl. J. Med. 314, 926-927. 
522 Steenland, K., Hein, M.J., Cassinelli, R.T. 2nd, Prince, M.M., Nilsen, N.B., Whelan, E.A. Waters 523 MA, Ruder AM, Schnorr TM., 2006. Polychlorinated biphenyls and neurodegenerative disease 524 mortality in an occupational cohort. Epidemiology. 17, 8-13.

Taylor, P.R., Lawrence, C.E., 1992. Polychlorinated biphenyls: estimated serum half-lives. Brit. 527 J. Ind. Med. 49, 527-528.

Webb, R.G., McCall, A.C., 1973. Quatititative PCB standards for electron capture gas

Wolff MS, Fischbein A, Selikoff IJ. Changes in PCB serum concentrations among capacitor 533 manufacturing workers. Environ Res 1992;59:202-16. 